\title{
Modelagem da ingestão, retenção e excreção de nitrogênio e fósforo pela suinocultura gaúcha: interface vegetal
}

\author{
Ingestion, retention and excretion modeling of nitrogen and phosphorus by pigs \\ in Rio Grande do Sul, Brazil: vegetable interface
}

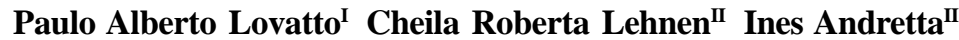 \\ Gustavo Dias Lovato ${ }^{\text {III }}$ Luciano Hauschild ${ }^{\text {II }}$
}

\section{RESUMO}

Um modelo foi desenvolvido com o objetivo de simular as dinâmicas de Nitrogênio e Fósforo pela suinocultura gaúcha associadas às produções de milho e soja do Rio Grande do Sul. O modelo é constituído por um submodelo animal (SMA) e outro vegetal (SMV). Os princípios gerais do SMA foram baseados em sistemas tecnológicos (ST), categorias animais, concentrações de $N$ e P das dietas e suas eficiências digestiva e metabólica. O SMA integra três níveis de agregação, sendo constituído de 10 compartimentos para $N$ e $P$ e 300 subcompartimentos relacionados aos ST e às categorias animais. Os dados da suinocultura gaúcha foram divididos em sete mesorregiões de acordo com as definições fisiográficas. Os ST foram definidos pelo número de suínos terminados/porca/ano. O SMV é constituído pelos subcompartimentos milho e soja. Os parâmetros do SMV foram baseados nas áreas cultivadas e nas produções de milho e soja de cada município. De acordo com o balanço entre a produção e o consumo, todas as mesorregiões possuem excedente de milho e soja. O consumo anual de milho $e$ farelo de soja pela suinocultura gaucha é em média de 1,14 e 0,39 milhões de toneladas, respectivamente. $O$ volume anual de $N$ e $P$ ingeridos pelos suínos é de aproximadamente 36 e 5,7 mil toneladas. Desse volume, cerca de $41 \%$ do $N$ e $71 \%$ do $P$ retornam ao meio ambiente. Os valores simulados dos diferentes cenários de oferta/demanda de milho e soja no Rio Grande do Sul mostram autosuficiência das sete mesorregiões. Os fluxos de nutrientes entre solo-planta-animal-solo simulados mostram que as mesorregiões do Rio Grande do Sul têm níveis de nitrogênio e fósforo lançados ao meio ambiente pelos suínos abaixo dos limites determinados pela União Europeia. O modelo simula adequadamente as transferências de N e P entre as interfaces animal e vegetal na produção suína, no Rio Grande do Sul.
Palavras-chave: fluxo de minerais, milho, nutrição, farelo de soja, suínos.

\section{ABSTRACT}

A model was developed to simulate the Nitrogen and Phosphorus dynamics of pigs in the Rio Grande do Sul state, Brazil associated with corn and soybean production. The model is constituted by an animal sub-model (SMA) and a vegetable one (SMV). The general approaches of SMA are based on technological systems (ST), animal categories, $N$ and $P$ concentrations in diets and its digestive and metabolic efficiencies. The SMA integrates three aggregation levels, constituted by ten compartments for $N$ and $P$ and 300 subcompartments related to ST and animal categories. The pig production data were divided in seven mesorregions in agreement with the physiographic definitions. The SMV is constituted by corn and soybean sub-compartments. The SMV parameters were based on the cultivated area and in the corn and soybean production of each municipal district. The simulated data show a surplus of corn and soybean in all mesorregions. Annual consumption of corn and soybean meal by pigs on the state is nearly to 1.14 and 0.39 million tons, respectively. Annual volume intake of $N$ and $P$ by the pigs is nearly 36 and 5.7 thousand tons, which $41 \%$ of $N$ and $71 \%$ of $P$ return to the environment. The simulated values of the different offer/demand sceneries of corn and soybean in Rio Grande do Sul show self-sufficiency of seven mesorregions. The flows of nutrients among soil-plant-animal-soil simulated show that Rio Grande do Sul mesorregions has levels of nitrogen and phosphorus released into the environment by the pigs below the limits imposed by the European Union. The model simulates appropriately the transfers of $N$ and $P$ between animal and vegetable interfaces in pig production in Rio Grande do Sul.

IDepartamento de Zootecnia (DZ), Universidade Federal de Santa Maria (UFSM), 97105-900, Santa Maria, RS, Brasil. E-mail: lovatto@smail.ufsm.br. Autor para correspondência.

IIPrograma de Pós-graduação em Zootecnia, UFSM, Santa Maria, RS, Brasil.

IIICurso de Zootecnia, UFSM, Santa Maria, RS, Brasil. 
Key words: mineral flows, soybean meal, corn, nutrition, pigs.

\section{INTRODUÇÃO}

Um dos temas mais debatidos na produção agrícola atual é a necessidade de estudar, compreender e manipular os sistemas de produção com o objetivo de torná-los mais harmônicos com o homem, o meio ambiente e os animais. Esse pressuposto emerge da necessidade de entender a produção de forma sistêmica e se torna mais evidente na suinocultura, por ser responsabilizada muitas vezes por desequilíbrios entre os componentes dos sistemas de produção em que ela é explorada intensivamente (LOVATTO et al., 2005a).

No entanto, ao longo das últimas décadas, os estudos sobre a cadeia de produção suína privilegiaram os aspectos relacionados com o animal ou a alimentação. Para relacionar quali e quantitativamente esses dois aspectos, é fundamental sistematizar as informações disponíveis e simular diferentes situações. Para que isso seja possível, é necessário o desenvolvimento de modelos dinâmicos e mecanicistas que permitam avaliar simultaneamente os cenários animal e vegetal.

Nos aspectos relacionados aos animais, alguns modelos foram publicados em nível mundial (BASTIANELLI et al., 1996; RODRIGUEZ-CASO et al., 2006; WELLOCK et al., 2004) e no Brasil (LOVATTO et al., 2005a; LOVATTO et al., 2005b). No caso brasileiro, os modelos priorizaram os aspectos digestivos e metabólicos, dando ênfase aos balanços do nitrogênio e do fósforo. Além disso, esses modelos não incorporam a autosuficiência regional de alimentos e o retorno para o solo de nutrientes excretados pelas vias fecal e urinária.

Tendo em vista que as interfaces animal e vegetal são indissociáveis, se torna necessário integrálas por meio da modelagem animal. Essa integração permite estudar diferentes cenários de oferta/demanda de ingredientes e os fluxos de nutrientes entre soloplanta-animal-solo.

A associação entre a sistematização das informações sobre as dinâmicas do nitrogênio e fósforo com a produção vegetal tem limitado a qualidade da avaliação dos sistemas de produção de suínos em diferentes regiões geográficas. O objetivo deste trabalho é acrescentar ao modelo de ingestão, retenção e excreção de nitrogênio e fósforo pela suinocultura gaúcha (LOVATTO et al., 2005b) uma interface vegetal associada às produções de milho e soja no Rio Grande do Sul (RS). Isso permitirá quantificar regionalmente os fluxos de nutrientes entre solo-planta-animal-solo baseados na cadeia de producão suína.

\section{MATERIAL E MÉTODOS}

O modelo da ingestão de nitrogênio e fósforo pela suinocultura gaúcha - interface vegetal (MSUINP/RSve) foi desenvolvido no Setor de Suinocultura e no Grupo de Modelagem Animal (CNPq - Grupos de Pesquisa), ambos localizados no Departamento de Zootecnia da Universidade Federal de Santa Maria. O modelo é constituído pelos submodelos animal (SMA) e vegetal (SMV). O SMA integra as dinâmicas de ingestão e excreção de $\mathrm{N}$ e $\mathrm{P}$ pelos animais. A base espacial e temporal do SMA foi descrita por LOVATTO et al. (2005b). Resumidamente, esse modelo é constituído de 10 compartimentos (N e $\mathrm{P}$ ingeridos, $\mathrm{N}$ e $\mathrm{P}$ sistema gastrintestinal, $\mathrm{N}$ e $\mathrm{P}$ metabólicos, $\mathrm{N}$ e $\mathrm{P}$ fecais, $\mathrm{N}$ e $\mathrm{P}$ urinários) e 300 subcompartimentos (50 associados aos sistemas tecnológicos e 250 associados às categorias animais). Os parâmetros dos fluxos de $\mathrm{N}$ e $\mathrm{P}$ usados no modelo são os mesmos do modelo original proposto por CARTER \& CROMWELL (1998) e DOURMAD et al. (1999). Esses parâmetros foram mantidos, pois nos últimos 10 anos não houve modificações entre eficiências (digestiva e metabólica) e genética animal e dos alimentos utilizados que justificasse o uso de dados mais recentes.

Para inclusão no modelo, os dados globais da suinocultura gaúcha foram decompostos por mesorregiões de acordo com definições fisiográficas (Nordeste, Centro Oriental, Noroeste, Metropolitana de Porto Alegre, Centro Ocidental, Sudoeste e Sudeste). Para cada mesorregião, foram quantificados o rebanho total, as matrizes, os leitões produzidos e os animais abatidos (IBGE, 2007).

O SMV foi desenvolvido tendo por base o milho e a soja, os dois ingredientes mais importantes da alimentação de suínos no RS. O SMV foi assim definido: para cada município dentro da cada mesorregião, foram quantificadas as áreas cultivadas e as produções de milho e soja segundo o Levantamento Sistemático da Produção Agrícola (IBGE, 2007). A produtividade foi estimada a partir dessas duas informações. Os consumos de milho e soja foram estabelecidos por categoria, levando em conta os critérios descritos na SMA. Para ajustar a soja cultivada e o consumo de farelo de soja, foi utilizada a relação 1,00:0,75, que é a quantidade média de farelo obtida após a extração do óleo da soja (WIESE \& SNYDER, 1987). A excreção por hectare de $\mathrm{N}$ e $\mathrm{P}$ considerou apenas as áreas cultivadas para 
alimentação de suínos (baseados no consumo pelos animais). Por opção metodológica, os valores dos fluxos de $\mathrm{N}$ e $\mathrm{P}$ animal-solo correspondem unicamente às áreas cultivadas de milho e soja para atender a demanda de alimentação dos suínos e não as áreas totais cultivadas com as duas culturas no RS. O modelo foi desenvolvido com ajuda dos programas Excel e Modelmaker (MODELMAKER, 1999).

\section{RESULTADOS E DISCUSSÃO}

Os consumos simulados de milho e soja por categoria animal e por mesorregião são apresentados na tabela 1 . O rebanho suíno gaúcho consome anualmente cerca de 1,14 milhões de toneladas de milho, o que correspondente ao cultivo de $367 \mathrm{mil}$ hectares. Os animais em crescimento/terminação (25$115 \mathrm{~kg}$ PV) consomem aproximadamente 54\% desse volume. O consumo anual de farelo de soja pela suinocultura gaúcha é de aproximadamente 0,39 milhões de toneladas, o que corresponde ao cultivo de 223 mil ha de soja. A análise regionalizada do consumo mostra que a mesorregião Noroeste consome cerca de $60 \%$ do milho e do farelo de soja. Se adicionarmos as mesorregiões Nordeste e CentroOriental à Noroeste, o consumo desses dois ingredientes alcança quase $92 \%$ do total.

Os balanços simulados de produção e consumo de milho e soja distribuídos por mesorregião são apresentados na tabela 2. De maneira geral, os resultados simulados mostram que todas as mesorregiões possuem excedentes de milho e soja para o consumo dos suínos, mas com participação relativa diferente para cada ingrediente. A região CentroOriental é a que destina a maior fração de farelo de soja para alimentação de suínos, alcançando uma média anual de cerca de $40 \%$. Com relação ao milho, a mesorregião Metropolitana de Porto Alegre é a que destina a maior quantidade para alimentação dos suínos, alcançando igualmente cerca de 40\%. Um dado interessante é o reduzido comprometimento da produção de milho da mesorregião Noroeste com a alimentação de suínos, que permanece abaixo de $4 \%$. Isso se explica pelo elevado volume produzido desses grãos nessa região.

Os valores da transferência de $\mathrm{N}$ e $\mathrm{P}$ do milho/soja-suínos-solo são apresentados na tabela 3. $\mathrm{O}$ volume anual de $\mathrm{N}$ ingerido pelos suínos é de aproximadamente 36 mil t, sendo 27 mil t por intermédio do farelo de soja e 9 mil t pelo milho. Desse volume, cerca de $41 \%$ retornam ao meio ambiente, o que corresponde a uma excreção anual média teórica total de $20 \mathrm{~kg} \mathrm{ha}^{-1}$ (limitado a áreas cultivadas com milho e soja destinadas à suinocultura). Os resultados simulados mostram que a região de maior concentração de suínos não é aquela que concentra a maior excreção de $\mathrm{N}$ por área cultivada de milho e soja destinada à alimentação dos suínos.

O volume anual de $\mathrm{P}$ ingerido pelos suínos é de aproximadamente 5,7 mil t, sendo 2,5 mil por intermédio do farelo de soja e 3,2 mil pelo milho. Desse volume, cerca de $71 \%$ retornam ao meio ambiente, o que corresponde a uma excreção anual média teórica de $5 \mathrm{~kg} \mathrm{ha}^{-1}$ (limitado a áreas cultivadas com milho e soja destinadas à suinocultura).

Um aspecto que deve ser considerado na transferência de $\mathrm{N}$ animal-solo é a volatilização da amônia ainda na instalação onde os animais estão alojados, que pode alcançar $6,8 \mathrm{~g} \mathrm{~d}^{-1}$ suíno $^{-1}$ (AARNINK \& ELZING, 1998) ou 3,7 kg ano-1 suíno $^{-1}$ terminado (DOORN et al., 2002). Outro aspecto que deve ser considerado é que as perdas continuam depois da aplicação no solo, e a aplicação pode permanecer, após 11 dias, em torno de 14,5\% do N aplicado (DUARTE et al., 2007). Essa volatilização depende da forma de aplicação, podendo alcançar $68 \%$ se for superficial, $17 \%$ se incorporado e $2 \%$ se injetado profundamente no solo (SØGAARD et al., 2002,

Tabela 1 - Consumos anuais (t x1000) simulados de milho e soja por suínos separados por categoria e mesorregião.

\begin{tabular}{|c|c|c|c|c|c|c|c|c|}
\hline \multirow{2}{*}{ Mesorregião } & \multicolumn{2}{|c|}{-------Gest/Lact---------- } & \multicolumn{2}{|c|}{--------Creche--------- } & \multicolumn{2}{|c|}{--------Crescimento-------- } & \multicolumn{2}{|c|}{------Terminação------ } \\
\hline & Milho & Soja & Milho & Soja & Milho & Soja & Milho & Soja \\
\hline Noroeste & 114,0 & 38,7 & 69,8 & 27,9 & 127,2 & 54,4 & 385,8 & 117,7 \\
\hline Centro Or. & 30,2 & 9,9 & 18,5 & 7,4 & 34,4 & 14,7 & 101,3 & 30,9 \\
\hline Nordeste & 27,9 & 8,9 & 17,7 & 7,1 & 33,3 & 14,2 & 91,1 & 27,8 \\
\hline Centro Oc. & 10,7 & 3,5 & 4,1 & 1,6 & 7,2 & 3,1 & 22,3 & 6,8 \\
\hline Metr. POA & 9,6 & 3,2 & 1,6 & 0,6 & 2,8 & 1,2 & 8,5 & 2,6 \\
\hline Sudeste & 6,6 & 2,2 & 0,9 & 0,3 & 1,6 & 0,7 & 5,5 & 1,7 \\
\hline Sudoeste & 4,9 & 1,6 & 0,9 & 0,3 & 1,6 & 0,7 & 5,2 & 1,6 \\
\hline Total & 203,9 & 68 & 113,5 & 45,2 & 208,1 & 89 & 619,7 & 189,1 \\
\hline
\end{tabular}


Tabela 2 - Balanços simulados anuais (t x1000) de produção e consumo de milho e soja no RS distribuídos por mesorregião.

\begin{tabular}{|c|c|c|c|c|c|c|}
\hline \multirow{2}{*}{ Mesorregião } & \multicolumn{3}{|c|}{--Milho----------------------------. } & \multicolumn{3}{|c|}{ 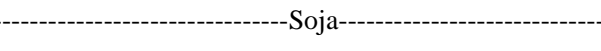 } \\
\hline & Produção & Consumo & Balanço & Produção & Consumo & Balanço \\
\hline Noroeste & 3.030 & 697 & 2.333 & 6.554 & 238 & 6.316 \\
\hline Nordeste & 668 & 169 & 499 & 222 & 58 & 164 \\
\hline Centro Or. & 464 & 184 & 280 & 216 & 63 & 153 \\
\hline Sudeste & 306 & 15 & 291 & 114 & 5 & 109 \\
\hline Centro Oc. & 203 & 44 & 159 & 881 & 15 & 866 \\
\hline Metr. POA & 146 & 22 & 124 & 19 & 7 & 12 \\
\hline Sudoeste & 105 & 13 & 92 & 470 & 4 & 466 \\
\hline Total & 4.922 & 1.144 & 3.778 & 8.476 & 390 & 8.086 \\
\hline
\end{tabular}

HUIJSMANS et al., 2003). Esses resultados mostram que, embora variáveis, a volatilização do $\mathrm{N}$ excretado pelos suínos é elevada. Essa perda também é observada quando se utiliza a cama de suínos em comparação aos dejetos líquidos ou à ureia (GIACOMINI \& AITA, 2008). Dessa forma, o máximo de $\mathrm{N}$ com potencial agronômico presente nas excretas de suínos não representa mais que 5\% das recomendações da Comissão Europeia, que limitam a aplicação em $170 \mathrm{~kg} \mathrm{ha}^{-1}$ ano $^{-1}$ (OENEMA, 2004, OENEMA et al., 2004). No caso brasileiro, a Resolução 377 do CONAMA, que normatiza a Lei da Política Nacional dos Recursos Hídricos, estabelece que o valor de nitrogênio total não deve ultrapassar 1,27mg $\mathrm{L}^{-1}$ para ambientes lênticos e $2,18 \mathrm{mg} \mathrm{L}^{-1}$ para ambientes lóticos (CONAMA, 2005). No entanto, a legislação em vigor não estabelece normativas que limitem a lançamento de nitrogênio nos solos brasileiros.

Os volumes de fósforo superiores a $20 \mathrm{~kg}$ $\mathrm{ha}^{-1}$ ano no solo estão normalmente associados com a produção animal intensiva (EDWARDS \& WITHERS, 1998). As mesorregiões Nordeste, Noroeste e Centro Oriental podem ser classificadas dentro desse conceito de produção. É importante ressaltar aqui a relatividade dos dados, pois nessas mesorregiões existem outras espécies criadas intensivamente que contribuem igualmente para o aumento do volume de fósforo lançado no meio ambiente. No entanto, esses níveis estão abaixo das recomendações da União Europeia, que limita em $80 \mathrm{~kg}$ de fósforo ha-1 ${ }^{-1} \mathrm{ano}^{-1}$ (TAMMINGA, 2003).

Para o cultivo de milho em sistema convencional, é recomendada uma aplicação média de $\mathrm{N}$ de $20 \mathrm{~kg} \mathrm{ha}^{-1}$ e de $\mathrm{P}_{2} \mathrm{O}_{5}$ em torno de $45 \mathrm{~kg} \mathrm{ha}^{-1}$ (ROLAS, 2004). Já para o cultivo da soja as recomendações variam de acordo com as situações de solo, os antecedentes culturais e o clima, podendo variar de 40 a $160 \mathrm{~kg} \mathrm{ha}^{-1}$ sem afetar a produtividade (MARTINAZZO et al., 2007). Se for considerada a média anual de retorno animal-solo de $5 \mathrm{~kg} \mathrm{ha}^{-1}$, esse valor alcança pouco mais de $10 \%$ da recomendação para o cultivo da soja.

De maneira geral, os resultados simulados mostram que as diferentes mesorregiões do Rio Grande do Sul são autossuficientes na produção de milho e soja para alimentação de suínos. Além disso, as

Tabela 3 - Transferências simuladas anuais de nitrogênio e fósforo vegetal-animal-solo por mesorregião.

\begin{tabular}{|c|c|c|c|c|c|c|c|c|}
\hline \multirow{3}{*}{ Mesorregião } & \multirow{2}{*}{\multicolumn{2}{|c|}{ Milho }} & \multirow{2}{*}{\multicolumn{2}{|c|}{ Soja }} & \multirow{2}{*}{\multicolumn{2}{|c|}{-------Excreção, t------- }} & \multicolumn{2}{|c|}{----Excreção, kg ha-1---- } \\
\hline & & & & & & & & \\
\hline & $\mathrm{N}$ & $\mathrm{P}$ & $\mathrm{N}$ & $\mathrm{P}$ & $\mathrm{N}$ & $\mathrm{P}$ & $\mathrm{N}$ & $\mathrm{P}$ \\
\hline Noroeste & 3.157 & 1.951 & 16.661 & 1.545 & 8.064 & 2.471 & 23 & 7 \\
\hline Centro Or. & 2.448 & 516 & 4.413 & 409 & 2.860 & 692 & 19 & 5 \\
\hline Nordeste & 2.245 & 473 & 4.066 & 377 & 2.605 & 632 & 27 & 6 \\
\hline Centro Oc. & 588 & 124 & 1.055 & 98 & 589 & 141 & 22 & 5 \\
\hline Metr. POA & 298 & 63 & 531 & 49 & 329 & 76 & 13 & 3 \\
\hline Sudoeste & 168 & 35 & 298 & 28 & 166 & 39 & 18 & 4 \\
\hline Sudeste & 194 & 41 & 344 & 32 & 214 & 49 & 20 & 4 \\
\hline Total & 9.098 & 3.203 & 27.368 & 2.538 & 14.827 & 4.100 & $20 *$ & $5 *$ \\
\hline
\end{tabular}

*média

Ciência Rural, v.40, n.4, abr, 2010. 
transferências de N e P animal-solo são baixas, tendo em vista as atuais recomendações de adubação para as culturas de soja e milho.

\section{CONCLUSÃO}

Os valores simulados dos diferentes cenários de oferta/demanda de milho e soja no Rio Grande do Sul mostram autossuficiência das sete mesorregiões. Os fluxos entre animal-solo simulados mostram que as mesorregiões do Rio Grande do Sul têm níveis de nitrogênio e fósforo lançados pelos suínos ao meio ambiente que apresentam valores médios inferiores aos limites impostos pela União Europeia. A transferência animal-solo de nitrogênio e fósforo, considerando as áreas cultivadas de milho e soja para suprir a demanda da suinocultura, corresponde respectivamente a 12 e $10 \%$ em valores médios e totais das recomendações de adubação.

\section{AGRADECIMENTOS}

Os autores agradecem à Comissão de Aperfeiçoamento de Pessoal de Nível Superior (CAPES), pelas bolsas de doutorado concedidas aos autores Cheila Roberta Lehnen e Luciano Hauschild e pela bolsa de mestrado concedida à autora Ines Andretta; ao Conselho Nacional para o Desenvolvimento Científico e Tecnológico (CNPq), pela bolsa de Produtividade em Pesquisa de Paulo Alberto Lovatto; e ao Fundo de Incentivo à Pesquisa da UFSM (FIPE/UFSM), pela bolsa concedida a Gustavo Dias Lovato. O presente trabalho, por utilizar uma abordagem meta-analítica, não necessita de parecer da Comissão de Ética para sua realização.

\section{REFERÊNCIAS}

AARNINK, A.J.A.; ELZING, A. Dynamic model for ammonia volatilization in housing with partially slatted floors, for fattening pigs. Livestock Production Science, v.53, p.153169, 1998. Disponível em: <http://dx.doi.org/10.1016/S03016226(97)00153-X>. Acesso em: 16 jun. 2009. doi: 10.1016/ S0301-6226(97)00153-X.

BASTIANELLI, D. et al. Mathematical modeling of digestion and nutrient absorption in pigs. Journal of Animal Science, v.74, p.1873-1887, 1996.

CARTER, S.D.; CROMWELL, G.L. Influence of porcine somatotropin on the phosphorus requirement of finishing pigs: I. Performance and bone characteristics. Journal of Animal Science, v.76, p.584-595, 1998. Disponível em: $<$ http://dx.doi.org/. Acesso em: 16 jun. 2009.

CONAMA. 2005. In: CONSELHO NACIONAL DO MEIO AMBIENTE (Ed.). Resolução n.377. Brasília: Ministério do Meio Ambiente, 2005. p.23.

DOORN, M.R.J. et al. Development of an emission factor for ammonia emissions from US swine farms based on field tests and application of a mass balance method. Atmospheric
Environment, v.36, p.5619-5625, 2002. Disponível em: <http://dx.doi.org/10.1016/S1352-2310(02)00689-1>. Acesso em: 16 jun. 2009. doi: 10.1016/S1352-2310(02)00689-1.

DOURMAD, J.Y. et al. Nitrogen and phosphorus consumption, utilisation and losses in pig production: France. Livestock Production Science, v.58, p.199-211, 1999. Disponível em: <http://dx.doi.org/10.1016/S0301-6226(99)00009-3>. Acesso em: 16 jun. 2009. doi: 10.1016/S0301-6226(99)00009-3.

DUARTE, F.M. et al. Perdas de nitrogênio por volatilização de amônia com aplicação de uréia em solo de várzea com diferentes níveis de umidade. Ciência Rural, v.37, p.705711, 2007. Disponível em: <http://dx.doi.org/10.1590/S010384782007000300016>. Acesso em: 16 jun. 2009. doi: 10.1590/S0103-84782007000300016

EDWARDS, A.C.; WITHERS, P.J.A. Soil phosphorus management and water quality. Soil use and Management, v.14, p.124-130, 1998. Disponível em: <http://dx.doi.org/ 10.1111/J.1475-2743.1998.TB00630.X>. Acesso em: 16 jun. 2009. doi: 10.1111/J.1475-2743.1998.TB00630.X.

GIACOMINI, S.J.; AITA, C. Cama sobreposta e dejetos líquidos de suínos como fonte de nitrogênio ao milho. Revista Brasileira de Ciência do Solo, v.32, p.195-205, 2008. Disponível em: <http://dx.doi.org/10.1590/S010006832008000100019>. Acesso em: 16 jun. 2009. doi: 10.1590/S0100-06832008000100019.

HUIJSMANS, J.F.M. et al. Effect of application method, manure characteristics, weather and field conditions on ammonia volatilization from manure applied to arable land. Atmospheric Environment, v.37, p.3669-3680, 2003. Disponível em: <http://dx.doi.org/10.1016/S13522310(03)00450-3>. Acesso em: 16 jun. 2009. doi: 10.1016/ S1352-2310(03)00450-3.

IBGE. Censos agropecuários: produção agrícola municipal. Cereais, leguminosas e oleaginosas anuais. Rio de Janeiro: Instituto Brasileiro de Geografia e Estatística, 2007. 146p.

LOVATTO, P.A. et al. Modelagem da ingestão, retenção e excreção de nitrogênio e fósforo pela suinocultura brasileira. Revista Brasileira de Zootecnia, v.34, p.2348-2354, 2005a. Disponível em: <http://dx.doi.org/10.1590/S151635982005000700022>. Acesso em: 16 jun. 2009. doi: 10.1590/S1516-35982005000700022.

LOVATTO, P.A. et al. Modelagem da ingestão, retenção e excreção de nitrogênio e fósforo pela suinocultura gaúcha. Ciência Rural, v.35, p.883-890, 2005b. Disponível em: <http://dx.doi.org/10.1590/S0103-84782005000400021>. Acesso em: 16 jun. 2009. doi: 10.1590/S010384782005000400021 .

MARTINAZZO, R. et al. Fósforo microbiano do solo sob sistema plantio direto em resposta à adição de fosfato solúvel. Revista Brasileira de Ciência do Solo, v.31, p.563-570, 2007. Disponível em: <http://dx.doi.org/10.1590/S010006832007000300016>. Acesso em: 16 jun. 2009. doi: 10.1590/S0100-06832007000300016.

MODELMAKER. ModelMaker. 3.0.4. Oxford, UK: Cherwell Scientific Publishing, 1999. 330p. 
OENEMA, O. Governmental policies and measures regulating nitrogen and phosphorus from animal manure in European agriculture. Journal of Animal Science, v.82, E-Suppl, p.E196-206, 2004. Disponível em: <http://dx.doi.org/. Acesso em: 16 jun. 2009.

OENEMA, O. et al. Environmental effects of manure policy options in The Netherlands. Water Science and Technology, v.49, p.101-108, 2004. Disponível em: <http:/ /dx.doi.org/. Acesso em: 16 jun. 2009.

RODRIGUEZ-CASO, C. et al. Mathematical modeling of polyamine metabolism in mammals. Journal Biology \& Chemistry, v.281, p.21799-21812, 2006. Disponível em: <http://dx.doi.org/10.1074/jbc.M602756200>. Acesso em: 16 jun. 2009 . doi: 10.1074/jbc.M602756200.

SØGAARD, H.T. et al. Ammonia volatilization from fieldapplied animal slurry-the ALFAM model. Atmospheric Environment, v.36, p.3309-3319, 2002. Disponível em: <http:/ /dx.doi.org/10.1016/S1352-2310(02)00300-X>. Acesso em: 16 jun. 2009. doi: 10.1016/S1352-2310(02)00300-X.
ROLAS. Recomendação de adubação e calagem para os estados do Rio Grande do Sul e Santa Catarina. 10.ed. Porto Alegre: SBCS, 2004. 400p.

TAMMINGA, S. Pollution due to nutrient losses and its control in European animal production. Livestock Production Science, v.84, p.101-111, 2003. Disponível em: <http://dx.doi.org/10.1016/j.livprodsci.2003.09.008>. Acesso em: 16 jun. 2009. doi: 10.1016/ j.livprodsci.2003.09.008.

WELLOCK, I.J. et al. Modeling the effects of stressors on the performance of populations of pigs. Journal of Animal Science, v.82, p.2442-2450, 2004. Disponível em: <http:// dx.doi.org/. Acesso em: 16 jun. 2009.

WIESE, K.L.; SNYDER, H.E. Analysis of the oil extraction process in soybeans: a new continuous procedure. Journal of the American Oil Chemists' Society, v.64, p.402-406, 1987. Disponível em: <http://dx.doi.org/10.1007/ BF02549304>. Acesso em: 16 jun. 2009. doi: 10.1007/ BF02549304. 